\title{
IL CONTROLLO NEUROENDOCRINO DEL COMPORTAMENTO ALIMENTARE
}

\author{
FRANCESCO CAVAGNINI (*)
}

\begin{abstract}
SunTO. - L'appetito è sottoposto ad un complesso sistema di regolazione che si esplica attraverso una serie di segnali centrali e periferici che interagiscono tra loro modulando il comportamento alimentare in base alle necessità individuali: condizioni contingenti (digiuno o recente introduzione di cibo) e stato nutrizionale generale (adiposità oppure magrezza). La regolazione periferica si realizza per mezzo di segnali che vengono definiti di adiposità e di sazietà, mentre il controllo centrale si effettua mediante una serie di effettori che comprendono il sistema neuropeptidergico, il sistema monoaminergico e il sistema endocannabinoide. I segnali di adiposità informano il cervello sullo stato nutrizionale generale dell'individuo, indicato dall'entità dei depositi adiposi. La leptina, prodotta dal tessuto adiposo e l'insulina, secreta dal pancreas endocrino in misura tendenzialmente crescente con l'espandersi della massa grassa, portano al cervello un segnale anoressante. I segnali di sazietà, che comprendono colecistochinica (CCK), glucagon-like peptide-1 (GLP-1) e peptide YY (PYY), originano nel tratto gastroenterico durante il passaggio del cibo e raggiungono il nucleo del tratto solitario (NTS), nel tronco encefalico caudale, attraverso fibre ascendenti del nervo vago. Dal NTS partono afferenze che proiettano al nucleo arcuato (ARC) dell'ipotalamo. Qui i segnali di sazietà vengono integrati con i segnali di adiposità e con altri segnali di provenienza ipotalamica e sovraipotalamica, così da formare una complessa rete di circuiti nervosi in grado di elaborare la risposta alimentare più appropriata alle esigenze nutrizionali. In particolare, nell'ARC vengono prodotti numerosi neuropeptidi con azione oressante quali neuropeptide Y (NPY) e agouti-related peptide (AGRP), o anoressante come pro-opiomelanocortina (POMC) e cocaine- and amphetamine-regulated transcript (CART). Più a valle dell'ARC si trovano altre aree cerebrali coinvolte nella regolazione del comportamento alimentare: tra queste, il nucleo paraventricolare (PVN), che secerne peptidi anoressanti come il thyrotropin-releasing hormone $(\mathrm{TRH})$, il corticotrophin-releasing hormone $(\mathrm{CRH})$ e l'ossitocina, l'area ipotalamica laterale (LHA) e l'area perifornicale (PFA), che producono le sostanze oressanti oressina A (OXA) e ormone concentrante la melanina $(\mathrm{MCH})$. In tempi più recenti, un grande interesse si è concentrato sul sistema degli
\end{abstract}

(*) Laboratorio di Ricerche in Neuroendocrinologia, Istituto Auxologico Italiano, Milano, Italia. E-mail: cavagnini@auxologico.it 
endocannabinoidi endogeni che svolgono sicuramente un ruolo importante, anche se non ancora del tutto chiarito, nel controllo del comportamento alimentare e del metabolismo energetico. In questo contesto, infine, si stanno accumulando crescenti evidenze sperimentali di un ruolo svolto dal microbiota intestinale, quel trilione di batteri che popola il tratto gastroenterico.

Dalla complessa interazione tra organi periferici e sistema nervoso centrale è nato il concetto di gut-brain-axis, ormai entrato a far parte della fisiologia. Una maggiore comprensione dei meccanismi che regolano il comportamento alimentare potrà consentire lo sviluppo di farmaci in grado di ridurre o aumentare il consumo di cibo.

ABSTRACT. - Appetite is regulated by a complex system of central and peripheral signals that interact in order to modulate eating behavior according the individual needs, i.e. the fasting or fed condition and the general nutritional status. Peripheral regulation includes adiposity signals and satiety signals, while central control is accomplished by several effectors, including the neuropeptidergic, monoaminergic and endocannabinoid systems. Adiposity signals inform the brain of the general nutritional status of the subject as indicated by the extent of fat depots. Indeed, leptin produced by the adipose tissue and insulin, whose pancreatic secretion tends to increase with the increase of fat mass, convey to the brain an anorexigenic message. Satiety signals, including cholecystokinin (CCK), glucagon-like peptide-1 (GLP-1) and peptide YY (PYY), originate from the gastrointestinal tract during a meal and, through the vagus nerve, reach the nucleus tractus solitarius (NTS) in the caudal brainstem. From NTS afferents fibers project to the arcuate nucleus (ARC) of the hypothalamus, where satiety signals are integrated with adiposity signals and with several hypothalamic and supra-hypothalamic inputs, thus creating a complex network of neural circuits that finally elaborate the most appropriate response, in terms of eating behavior. In more detail, ARC neurons secrete a number of neuropeptides with orexigenic properties, such as neuropeptide Y (NPY) and agouti-related peptide (AGRP), or anorexigenic effects such as pro-opiomelanocortin (POMC) and cocaine- and amphetamine-regulated transcript (CART). Other brain areas involved in the control of food intake are located downstream the ARC: among these, the paraventricular nucleus (PVN), which produces anorexigenic peptides such as thyrotropin releasing hormone (TRH), corticotrophin releasing hormone $(\mathrm{CRH})$ and oxytocin, the lateral hypothalamus (LHA) and perifornical area (PFA), secreting the orexigenic substances orexin-A (OXA) and melanin concentrating hormone $(\mathrm{MCH})$. Recently, a great interest has developed for endogenous cannabinoids, important players in the regulation of food intake and energy metabolism. In the same context, increasing evidence is accumulating for a role played by the microbiota, the trillion of microorganism populating the human gastrointestinal tract.

The complex interaction between the peripheral organs and the central nervous system has generated the concept of gut-brain axis, now incorporated into the physiology. A better understanding of the mechanisms governing the eating behavior will allow the development of drugs capable of reducing or enhancing food consumption. 
Il cibo, insieme all'ossigeno dell'aria che ne permette l'utilizzazione, è indispensabile per la sopravvivenza delle specie animali, e distorsioni del suo consumo, in eccesso o in difetto, portano allo sviluppo di condizioni patologiche. Non sorprende quindi che in milioni di anni di evoluzione, l'organismo umano abbia sviluppato sofisticati meccanismi di controllo del comportamento alimentare diretti a mantenere l'equilibrio energetico. Si tratta di meccanismi influenzati da fattori ambientali, culturali, psicologici e da fattori endogeni di tipo squisitamente neuroendocrino. Questi ultimi, come dice il termine, sono costituiti da ormoni e da neurotrasmettitori che a livello del sistema nervoso centrale modulano il senso di fame e di sazietà e modificano il dispendo energetico.

Fino a metà del secolo scorso si riteneva che due aree cerebrali, il nucleo ventromediale e l'area laterale dell'ipotalamo, fossero i responsabili rispettivamente della sazietà e della fame. Nell'animale da esperimento, infatti, la lesione del primo e la stimolazione del secondo conducevano ad iperfagia e a sviluppo di obesità. Gli ultimi decenni hanno permesso di comprendere come il controllo del comportamento alimentare sia un fenomeno molto più articolato, che vede in gioco numerose componenti e presenta un altissimo grado di integrazione (1-3). In questo complesso sistema è possibile riconoscere un controllo periferico ed un controllo centrale.

La regolazione periferica ha il compito di far pervenire a specifiche aree cerebrali due tipi essenziali di informazioni: a) quale è lo stato di nutrizione generale dell'organismo, espresso dalla quantità di tessuto adiposo in esso presente b) quale è lo stato di nutrizione contingente, espresso da quanto (e anche da ciò che) il soggetto sta mangiando o ha da poco terminato di mangiare. Sulla base di tali informazioni il cervello, e segnatamente l'ipotalamo, attiva il controllo centrale che, grazie all'azione concertata di diversi centri, è in grado di fornire la risposta appropriata. Infatti, da queste aree cerebrali verranno rilasciate sostanze in grado di stimolare la sazietà e inibire la fame se l'individuo è in buono stato di nutrizione (soggetto sovrappeso e/o che ha appena consumato un pasto) o di indurre l'effetto opposto se il soggetto è denutrito e/o digiuno. In pratica, a seconda delle necessità, vengono accesi circuiti catabolici (diretti a bloccare l'ulteriore assunzione di cibo e a smaltire l'eccesso di energia introdotta) o anabolici (diretti a favorire l'assunzione di cibo e a conservare l'energia introdotta) (Fig. 1). 


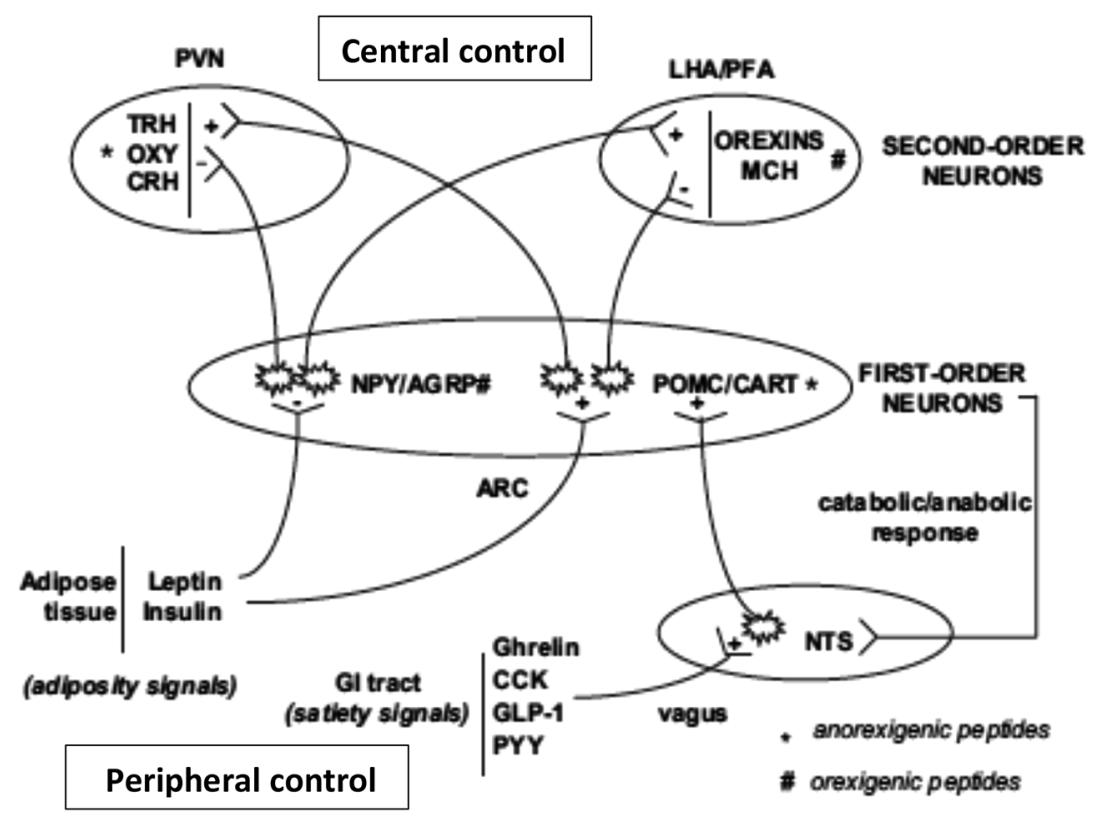

Fig. 1. Rappresentazione schematica dei circuiti cerebrali che presiedono alla regolazione del comportamento alimentare. ARC, nucleo arcuato; NTS, nucleo tratto solitario; CCK, colecistochinina; GLP-1, glucagon-like peptide 1; PYY, peptide YY; PVN, nucleo paraventricolare; $L H A$, area ipotalamica laterale; PFA, area perifornicale; NPY, neuropeptide Y; AGRP, Agouti-related peptide; POMC, proopiomelanocortina; CART, cocaine- and amphetamineregulated transcript; $C R H$, corticotropin-releasing bormone; TRH, thyrotropin-releasing bormone; $\mathrm{OX}$, ossitocina; $\mathrm{MCH}$, melanin-concentrating bormone (da Scacchi M. et al.).

\section{CONTROLLO PERIFERICO: SEGNALI SI ADIPOSITÀ E SEGNALI DI SAZIETÀ}

I segnali periferici che informano il cervello sullo stato di nutrizione generale provengono dal tessuto adiposo e sono per questo definiti segnali di adiposità. Merita qui precisare come negli ultimi decenni i concetti anatomo-funzionali del tessuto adiposo siano radicalmente cambiati al di là della distinzione già nota tra adipe bruno e adipe bianco, il primo scarso, profondo e deputato alla dispersione di energia in forma di calore (termogenesi), il secondo, più abbondante, sottocutaneo, con funzione di deposito di trigliceridi e quindi di energia. Oltre alla recente dimostrazione che il tessuto adiposo bruno può espandersi o ridursi, a seconda delle 
necessità, per trasformazione dal o nel tessuto adiposo bianco, oggi è ormai acquisita la nozione che il tessuto adiposo bianco, lungi dal rappresentare un mero deposito energia, sia dotato di una grande dinamicità metabolica. Esso è infatti in grado di produrre un elevato numero di sostanze, alcune con caratteristiche di ormoni (l'esempio più significativo è rappresentato dalla leptina), altre di natura enzimatica, altre ancora con attività pro-coagulativa o pro-infiammatoria. Considerata la natura citochinica o chemochinica di molte di queste sostanze e la sede della loro provenienza, è stato introdotto per definirle il termine comprensivo di adipochine (4). Queste possono agire localmente o, immesse in circolo, a distanza. Per queste sue caratteristiche, e per avere esso una distribuzione bene localizzata piuttosto che diffusa come si riteneva in passato, il tessuto adiposo viene oggi considerato alla stregua di un vero e proprio organo, l'organo adiposo appunto, e viene inoltre considerato un organo endocrino (5-8). Ai fini della presente trattazione, la principale adipochina prodotta dal tessuto adiposo bianco è la leptina (dal greco leptos $=$ snello, magro), un peptide di 167 aminoacidi, che costituisce il primo segnale di adiposità. La identificazione del gene della leptina nel 1994 (9) e in seguito del suo recettore, ha permesso di stabilire che questo peptide rappresenta il principale messaggio periferico che, attraverso il sangue, segnala all'ipotalamo lo stato di nutrizione generale dell'organismo. La leptina, prodotta in misura grosso modo proporzionale alla massa grassa, è dotata di potente azione anoressante oltre che stimolante il dispendio energetico: in questo modo essa genera un processo catabolico laddove lo stato di nutrizione è eccessivo, mentre nel caso contrario, con l'abbassamento dei suoi livelli, vengono favoriti i processi anabolici. Animali da laboratorio che mancano del gene della leptina (topi ob/ob) o che presentano una mutazione del suo recettore ( $\mathrm{ratti} \mathrm{fa} / \mathrm{fa}$ ) sviluppano precocemente iperfagia e grave obesità. Nell'uomo sono stati descritti rari casi con queste alterazioni nei quali, a conferma del ruolo della leptina, la somministrazione dell'ormone ha permesso una rapida e imponente perdita di adipe. Purtroppo, il sogno iniziale di poter curare l'obesità essenziale con la leptina è svanito con la constatazione che nell'individuo obeso le elevate concentrazioni circolanti di leptina non riescono a svolgere la dovuta azione anoressante per una concomitante resistenza all'azione del peptide. Della leptina va anche ricordato il suo ruolo importante, ma non discusso in questa sede, nel favorire la funzione riproduttiva (10-12).

Il secondo segnale di adiposità inviato dalla periferia all'ipotalamo è costituito dall'insulina, secreta dalla cellula beta del pancreas endocri- 
no e dotata, come la leptina, di azione anoressante. In presenza di riserve adipose esuberanti, l'eccesso di lipidi nell'organismo determina una resistenza all'azione dell'insulina, la cui secrezione va incontro ad un aumento di significato compensatorio. Leptina e insulina, giunte all'ipotalamo, innescano l'azione catabolica del controllo centrale $(13,14)$.

I segnali periferici sullo stato nutrizionale contingente provengono dall'intestino e vengono definiti segnali di sazietà. Già nello stomaco l'arrivo del cibo inibisce la secrezione di un ormone, la ghrelina, secreto da cellule del fondo gastrico e dotato di forte attività stimolante l'appetito (15). Allo stato attuale delle conoscenze, la ghrelina costituisce l'unico ormone periferico con azione oressizzante. I suoi livelli circolanti risultano elevati nel digiuno e nell'anoressia nervosa, mentre sono ridotti dopo il pasto e nell'obesità. Interessante ricordare che il fondo gastrico produce anche leptina, anche se l'importanza di questa secrezione resta ancora da stabilire. La stessa distensione gastrica dà origine ad impulsi nervosi che, attraverso rami dello splancnico e poi attraverso il nervo vago, raggiungono il tronco encefalico da cui irradiano all'ipotalamo e a centri encefalici superiori. La progressione del cibo verso le successive porzioni dell'intestino innesca, da parte di alcune cellule localizzate nella sua parete, la secrezione di una serie di ormoni che, per via ematica e nervosa vagale, trasmettono anch'essi informazioni al tronco encefalico e all'ipotalamo (16-19). Tra questi, la colecistochinina (CCK), il primo fattore anoressante intestinale ad essere identificato, è secreta dalle cellule I del duodeno e del digiuno in risposta all'ingestione di proteine e di grassi. Alcuni dati sperimentali indicherebbero che gli effetti sazianti delle fibre così come quello della Apolipoproteina A IV, rilasciata dal piccolo intestino in risposta al passaggio di grassi, siano mediati proprio dalla secrezione di CCK. L'effetto della CCK viene potenziato dalla leptina. Il passaggio del cibo nell'intestino induce anche la liberazione di gastric-inhibitory polypeptide o glucose-dependent insulinotropic polypeptide (GIP) e di Glucagon-like peptide-1 (GLP-1) da parte rispettivamente delle cellule intestinali K ed L. GIP e GLP-1 sono molecole peptidiche derivanti dal precursore del glucagone (pro-glucagone), in grado di favorire la sintesi-secrezione di insulina in risposta al glucosio, attenuando simultaneamente la secrezione del glucagone, di rallentare lo svuotamento gastrico e, importante ai nostri fini, di ridurre l'assunzione di cibo e quindi il peso corporeo. Questi peptidi, definiti incretine, agiscono in buona parte attraverso vie nervose vagali dotate di specifici recettori per queste molecole. Le azioni sopra citate rendono ragione dell'impiego di 
queste sostanze, di loro analoghi o di agonisti dei loro recettori, nel diabete di tipo 2 e del crescente interesse per un loro potenziale impiego nell'obesità. Quanto detto vale anche per alcuni inibitori dell'enzima dipeptidilpeptidasi IV, che presiede alla degradazione delle due incretine. È recente la dimostrazione di un aumento di densità delle cellule intestinali producenti GLP-1 dopo bypass gastrico, una osservazione che può spiegare il riscontro di aumentati livelli circolanti di questa incretina e del miglioramento del diabete dopo l'intervento (20). Dalle cellule intestinali $\mathrm{L}$ viene secreta un'altra sostanza, anch'essa derivante dal pro-glucagone e dotata di azione saziante, la oxintomodulina. In risposta al passaggio dei vari nutrienti, l'intestino secerne anche bombesina, neurotensina e peptide YY (PYY), sostanze tutte dotate di azione inibente l'appetito. Il PYY, anch'esso prodotto dalle cellule intestinali L, è il più potente tra i messaggi anoressanti inviati dagli enteroormoni. Nei soggetti obesi, i livelli circolanti postprandiali di PYY diminuiscono in misura minore di quanto si osserva nei soggetti normopeso e questo potrebbe anticipare in questi pazienti il desiderio del pasto successivo. Lo stesso comportamento si verifica per il GLP-1. Sebbene non si tratti di un enteroormone, va ricordata l'amilina, cosecreta con l'insulina dalle cellule pancreatiche, che nell'animale da esperimento riduce significativamente l'assunzione di cibo (21).

Tra i segnali periferici di sazietà è stato recentemente ipotizzato l'intervento di specifici aminoacidi derivanti dalla digestione delle proteine alimentari. Agendo a livello della mucosa intestinale e per via ematica, indurrebbero a livello del sistema nervoso centrale una risposta anoressante oltre che un rallentato svuotamento dello stomaco (22). Al termine di questa disamina, è interessante considerare come a fronte di un elevato numero di segnali di tipo anoressante vi sia un solo segnale, la ghrelina, di segno opposto. Questa osservazione sembra indicare che, per la salvaguardia della sopravvivenza, l'organismo sia primariamente programmato per favorire l'introduzione di cibo e sia dotato al tempo stesso di molteplici meccanismi diretti al suo contenimento.

\section{CONTROLLO CENTRALE}

È responsabile della risposta comportamentale e metabolica alle informazioni pervenute dal sistema di controllo periferico e si realizza a livello di specifiche aree ipotalamiche con la liberazione, a seconda 
delle esigenze nutrizionali, di peptidi con azione anoressante e stimolatoria sul metabolismo energetico oppure dotati di azione opposta. È facile rendersi conto della complessità di questo sistema di controllo, dovendo esso tenere conto dello stato nutrizionale generale (segnali di adiposità) e di quello momentaneo (segnali di sazietà) fornendo risposte perfettamente appropriate. Dopo un pasto verranno quindi attivati circuiti catabolici, diretti allo spegnimento della fame (o all'induzione di sazietà) e all'aumento del dispendio energetico, con contemporanea inibizione dei circuiti anabolici. Il processo contrario si verificherà nell'individuo a digiuno $(23-25)$. L'ipotalamo è la sede elettiva di questi eventi e al suo interno la prima stazione di ricezione dei messaggi periferici è rappresentata dal nucleo arcuato, al quale essi giungono direttamente o in via mediata dal nucleo tratto solitario situato nel tronco encefalico. Nel nucleo arcuato, neuroni di $1^{\circ}$ ordine rilasciano peptidi con azioni differenziate: neuropeptide Y (NPY) e Agouti-related peptide $(\mathrm{AgRP})$ con potente azione oressizzante e, con azione opposta, proopiomelanocortina (POMC) e il suo derivato -MSH o melanocortina $(\mathrm{MC})$, e cocaine and amphetamine-regulated peptide (CART). Le fibre dei neuroni di $1^{\circ}$ ordine proiettano a neuroni di $2^{\circ}$ ordine localizzati in altre aree: nucleo paraventricolare, area ipotalamica laterale, area perifornicale. Il primo di questi nuclei rilascia peptidi anoressanti, attivatori di vie cataboliche, quali corticotropin-releasing hormone (CRH), urocortina, proteina mogano, ossitocina $(\mathrm{OXY})$ e thyrotropic-releasing hormone (TRH). Le altre due aree avviano circuiti anabolici secernendo peptidi che stimolano l'assunzione di cibo quali oressine, melaninconcentrating hormone $(\mathrm{MCH})$ e oppioidi endogeni. La degradazione della POMC dà origine ad altre molecole tra cui la MC, importante regolatore negativo del bilancio energetico. L'attività dei neuroni POMC è stimolata dalla leptina, la cui azione anoressante è potenziata dalla simultanea inibizione dei neuroni NPY/AgRP. D'altra parte, i neuroni POMC vengono silenziati dai neuroni NPY/AgRP in via mediata dal neurotrasmettitore inibitorio gamma-amino-butirrico (GABA). Individui con mutazioni del gene della POMC, così come topi deficitari del peptide, sviluppano precocemente iperfagia e obesità. Si ritiene oggi che almeno l' $8 \%$ della obesità grave a comparsa precoce nell'uomo sia legata ad una mutazione del recettore 3 o 4 della MC. Diverse evidenze sperimentali dimostrano come AgRP agisca impedendo a MC di attivare il suo recettore. (26) Recentemente, tuttavia, è stato dimostrato che AgRP può indurre l'assunzione di cibo per via diretta, 
indipendente dalla sua azione inibitoria sulla $\mathrm{MC}$, anche se le vie nervose specifiche di questa azione non sono ancora bene identificate (27). Interessante ricordare l'intervento della MC sul metabolismo. La MC esercita una influenza positiva sui neuroni secernenti TRH e quindi sulla funzione tiroidea e sul dispendio energetico. In condizioni di digiuno, l'attivazione dei neuroni $\mathrm{AgRP}$, che inibiscono i neuroni $\mathrm{MC}$, determina un abbassamento dell'attività tiroidea assicurando una riduzione del dispendio energetico. Le strette relazioni anatomo-funzionali tra neuroni POMC/MC, neuroni NPY/AgRP e GABA, che vedono la melanocortina al centro di una via catabolica di primaria importanza, hanno portato al concetto di sistema melanocortinico ipotalamico. (28). Anche mutazioni del gene che codifica per CART sono associate a riduzione del dispendio energetico e sviluppo di obesità nell'uomo. $\mathrm{MC}$ e CART vengono stimolati dalla leptina e dall'insulina e inibiti dalla ghrelina $(29,30)$. NPY e AgRP, potenti peptidi anabolici, vengono in larga misura co-secreti dagli stessi neuroni nel nucleo arcuato, il loro rilascio è inibito da leptina e insulina e stimolato da ghrelina. Quest'ultima potenzia la propria azione oressizzante inibendo l'enzima prolil-carbossipeptidasi che degrada la POMC. Al contrario, bassi livelli circolanti di leptina e di insulina, così come bassi valori di glicemia, espressioni di bilancio energetico negativo, stimolano la sintesi di NPY nel nucleo arcuato. Molti esperimenti nell'animale da laboratorio hanno potuto dimostrare un forte aumento dell'introduzione di cibo insieme ad una riduzione della termogenesi, e quindi sviluppo di obesità, dopo iniezione intra-cerebro-ventricolare di NPY (31). Diverse molecole con attività antagonista dei recettori Y1 e Y5 di NPY sono allo studio come potenziali farmaci anti obesità. Recenti studi hanno messo in evidenza che NPY, agendo per via ematica e mediante il sistema nervoso simpatico, è anche in grado di esercitare un'azione regolatoria sul tessuto adiposo. In questa sede, coerentemente con la sua funzione anabolizzante, NPY promuove la differenziazione adipocitaria, favorisce l'accumulo di energia nell'adipocita e previene la trasformazione del tessuto adiposo bianco in tessuto adiposo bruno, noto dissipatore di energia (32). È presumibile che una simile attività periferica possa essere svolta da altri neuropeptidi cerebrali.

Tra i neuroni di $2^{\circ}$ ordine, meritano un commento particolare quelli del nucleo paraventricolare producenti CRH. Questo peptide, oltre ad essere dotato di potente azione anoressante, costituisce il motore dell'asse ipotalamo-ipofisi-surrene e della risposta allo stress. Grazie 
alla prima di queste azioni, esso allontana l'esigenza di procurarsi e consumare il cibo, consentendo la massima concentrazione sull'evento da fronteggiare. Al tempo stesso eleva lo stato di attenzione, aumenta l'attività motoria e la frequenza respiratoria e - di somma importanza - stimola la secrezione ipofisaria di ACTH e quindi quella surrenalica di cortisolo. Quest'ultimo, attraverso un'azione lipolitica e la produzione epatica di glucosio, fornisce all'organismo una immediata disponibilità di nutrienti che, insieme ad un aumento della pressione arteriosa e alle azioni ricordate più sopra, lo mette in condizioni ottimali per fronteggiare lo stato di emergenza $(33,34)$. Dal nucleo paraventricolare viene anche liberata ossitocina (OXY), soprattutto in risposta alla distensione gastrica e ad un aumento dell'osmolarità plasmatica. OXY induce sazietà e porta a terminare il pasto. È uno dei mediatori dell'effetto anoressante della MC e del GLP-1. Modelli animali con deprivazione di OXY sviluppano obesità e nei pazienti con sindrome di Prader-Willi, caratterizzata tra l'altro da estrema iperfagia e grave obesità, è stata descritta una riduzione dei neuroni producenti OXY (35). Questo neuropeptide interviene anche nel condizionare le preferenze per i macronutrienti e la gratificazione (reward) data dagli stessi. Attraverso questo meccanismo, OXY induce un minor consumo di carboidrati ed è attraverso una ridotta liberazione di OXY che gli oppioidi aumentano l'ingestione di cibo e la preferenza per i carboidrati $(36,37)$. Al complesso meccanismo di reward, che vede implicata in primo luogo la dopamina, prendono parte anche $\mathrm{MC}$ e $\mathrm{AgRP}(38)$. Neuroni di $2^{\circ}$ ordine dell'area ipotalamica laterale e perifornicale producono invece neuropeptidi con azione oressizzante e anabolica: le oressine o ipocretine e l'ormone concentrante la melanina $(\mathrm{MCH})$. Le oressine A e B, delle quali la prima sembra essere la più attiva, esercitano numerose azioni tra le quali l'influenza sulla condotta alimentare non appare la più significativa. Esse intervengono nella regolazione del ritmo sonno-veglia attraverso un aumento dello stato di vigilanza, influenzano in modo complesso il metabolismo glucidico (aumentano la produzione epatica di glucosio e la sua utilizzazione nel tessuto muscolare) e infine stimolano l'attività del sistema nervoso simpatico. Quest'ultima è probabilmente l'azione principale svolta da questi neuropeptidi, in larga parte responsabile dell'aumento della termogenesi, e quindi del dispendio energetico, che essi inducono ad opera del tessuto adiposo bruno. I loro effetti sulla introduzione di cibo sono alquanto variabili a seconda delle condizioni sperimentali. Nel ratto, ad esempio, l'iniezione intra-cerebro-ventricolare 
di oressina A aumenta il consumo di cibo se praticata nelle ore di luce ma non se effettuata subito prima delle ore di oscurità, suggerendo un effetto legato al ritmo sonno-veglia. Quest'ultimo sembra di fatto fortemente influenzato dalle oressine poichè sia nell'animale che nell'uomo un deficit di oressine è associato a narcolessia. È probabile che le oressine agiscano anche a livello dei tessuti periferici. Esse sono infatti misurabili nel sangue, anche se non è ancora chiaro se provengano dal sistema nervoso centrale o vengano sintetizzate alla periferia (39). Neuroni localizzati nelle stesse aree ipotalamiche producono $\mathrm{MCH}$, neuropeptide con spiccata azione anabolica in quanto stimola il consumo di cibo e, secondo alcune evidenze sperimentali, abbassa il metabolismo energetico. L'espressione di MCH a livello ipotalamico viene antagonizzata dalla leptina. Di fatto, $\mathrm{MCH}$ risulta sovraespresso in roditori a digiuno oppure obesi con deficit di leptina. Per contro, topi deficitari di $\mathrm{MCH}$ sono ipofagici e magri, e resistono all'incremento ponderale indotto dalla sovralimentazione (40-42). Il peptide, i cui recettori sono largamente distribuiti nel sistema nervoso centrale, è coinvolto in altre funzioni: appare infatti dotato di effetti ansiogeni e depressivi, interviene nella regolazione del sonno, promuovendo il sonno profondo e quello della fase REM, un'azione opposta a quella svolta dalle oressine; potrebbe inoltre favorire i processi di apprendimento e memorizzazione e infine interviene, attraverso il sistema dopaminergico mesolimbico, a rinforzare i meccanismi di gratificazione $(38,43)$. È facile comprendere come molecole antagoniste dei recettori di $\mathrm{MCH}$ possano rappresentare potenziali farmaci per il trattamento di diverse patologie oltre all'obesità (44).

La descrizione del sistema di controllo del comportamento alimentare e del metabolismo energetico qui riportata è una rappresentazione schematica e semplificata di un meccanismo assai più complesso e sofisticato e non ancora del tutto chiarito. Questo sistema è integrato dall'intervento di altri meccanismi modulatori che agiscono attraverso neurotrasmettitori (catecolamine, dopamina, serotonina), aminoacidi (acido gamma amino butirrico, glutammato e altri) e tramite molecole lipidiche di più recente identificazione come gli endocannabinoidi endogeni (anandamide, 2-arachidonoilglicerolo e altre). Tra i neurotrasmettitori, la serotonina è in grado di promuovere il senso di sazietà mentre la dopamina svolge un ruolo specifico nei processi di reward, rinforzando la gratificazione per certi macronutrienti (45). Tra gli aminoacidi, arginina, lisina e acido glutammico sembrano svolgere un ruolo 
preminente. Nel ratto essi sono stati recentemente riconosciuti come gli aminoacidi maggiormente dotati di azione anoressante e che pertanto spiegano meglio il potere saziante delle proteine alimentari. Essi inviano messaggi all'area postrema e al nucleo tratto solitario oltre a rallentare lo svuotamento gastrico, un'azione quest'ultima che tuttavia sembra ininfluente sull'effetto saziante (46). Gli endocannabinoidi endogeni, i loro recettori CB1 e CB2 e gli enzimi deputati alla loro sintesi e degradazione costituiscono il sistema endocannabinoide (SEC). Agiscono come neurotrasmettitori a livello sia cerebrale che periferico agendo sui neuroni di $1^{\circ}$ e $2^{\circ}$ ordine con effetto oressizzante. Recenti studi hanno evidenziato come essi intervengano anche sui recettori CB1 della parete intestinale influenzando negativamente il rilascio locale di segnali di sazietà. Ancora, stimolano la risposta di gratificazione, accentuando il desiderio per determinati cibi. Infine, studi con l'impiego di antagonisti del recettore CB1 portano a concludere che il SEC influenza anche il metabolismo riducendo la spesa energetica (47-50) e stimolando la lipolisi nel tessuto adiposo bianco.

Quanto fin qui riportato mette in luce la ridondanza dei meccanismi di controllo del comportamento alimentare, il che conferisce all'intero sistema enormi capacità di supplenza quando uno dei meccanismi venga alterato. Questo assetto, se da un lato rappresenta una garanzia di stabilità omeostatica, dall'altro può vanificare i tentativi farmacologici di modificare alcuni circuiti neuroendocrini, pregiudicando i risultati terapeutici.

Lo scenario della regolazione della condotta alimentare e del metabolismo energetico non sarebbe completo senza un accenno al microbiota $(51,52)$. Questo è costituito da un trilione di microorganismi, del peso stimato di 1-2 Kg, che popolano il nostro intestino. Un numero crescente di risultati sperimentali nell'animale e nell'uomo sta dimostrando in modo sempre più convincente che il microbiota contribuisce alla regolazione sopra ricordata influenzando la liberazione intestinale dei fattori di sazietà. Alcuni microorganismi producono GABA, il principale neurotrasmettitore inibitorio del sistema nervoso centrale, mentre altri sono in grado di produrre catecolamine. La flora batterica intestinale, a seconda della sua costituzione, può modificare alcune secrezioni endocrine, ad esempio la risposta dell'asse ipotalamo-ipofisisurrene allo stress, ed è a sua volta modificata da alcune secrezioni ormonali. Esso interviene inoltre, insieme ad altri fattori, nella patogenesi di alcune alterazioni che caratterizzano l'obesità e la sindrome 
metabolica, come la infiammazione cronica di basso grado. Per queste sue caratteristiche il microbiota viene da alcuni proposto come un organo virtuale e come organo endocrino.

La complessa interazione tra gli organi periferici dell'organismo e il sistema nervoso centrale configura il concetto di Brain-Gut Axis sviluppato negli ultimi decenni e ormai acquisito dalla fisiologia. Si tratta di un esempio mirabile di perfetta integrazione funzionale tra organi tra loro distanti, volta ad assicurare una continua omeostasi nutrizionale.

La conoscenza sempre più approfondita dei meccanismi che controllano il comportamento alimentare e la identificazione delle numerose sostanze in esso coinvolte potrà avere ricadute terapeutiche permettendo lo sviluppo farmacologico di molecole in grado di ridurre o stimolare l'assunzione di cibo.

\section{BIBLIOGRAFIA}

1. Valassi E, Scacchi M, Cavagnini F. Neuroendocrine control of food intake. Nutr Metab Cardiovasc Dis, 2008:18:158-68.

2. Asarian L, Bächler T. Neuroendocrine control of satiation. Horm Mol Biol Clin Investig, 2014: doi: 10.1515/hmbci-2014-0010.

3. Ulrich-Lai YM, Ryan KK. Neuroendocrine circuits governing energy balance and stress regulation: functional overlap and therapeutic implications. Cell Metab, 2014: 19: 910.925.

4. Trayhurn P, Wood IS. Adipokines: inflammation and the pleiotropic role of white adipose tissue. Br J Nutr, 2004: 92: 347-55.

5. Mohamed-Ali V, Pinkney JH, Coppack SW. Adipose tissue as an endocrine and paracrine organ. Int J Obes Relat Metab Disord, 1998: 22: 1145-58.

6. Cinti S. The adipose organ. Ed. Kurtis, Milano: 1999.

7. Kershaw EE, Flier JS. Adipose tissue as an endocrine organ. J Clin Endocrinol Metab, 2004: 89:2548-56.

8. Scherer PE. Adipose tissue: from lipid storage compartment to endocrine organ. Diabetes, 2006: 55: 1537-45.

9. Zhang Y, Proenca R, Maffei M, Barone M, Leopold L, Friedman JM. Positional cloning of the mouse obese gene and its human homologue. Nature, 1994: 372: 425-32

10. Chehab FF. 20 years of leptin: leptin and reproduction: past milestones, present undertakings, and future endeavors. Journal of Endocrinology, 2014: 223: T37-T48.

11. Farooqi IS, O'Rahilly S. 20 years of leptin: human disorders of leptin action. J Endocrinol, 2014:223:T63-70.

12. Friedman J. 20 years of leptin: leptin at 20: an overview. J Endocrinol, 2014: 223:T1-8.

13. Baskin DG, Figlewicz Lattemann D, Seeley RJ, Woods SC, Porte D, Jr., Schwartz 
MW. Insulin and leptin: dual adiposity signals to the brain for the regulation of food intake and body weight. Brain Res,1999: 848: 114-23.

14. Bruning JC, Gautam D, Burks DJ, et al. Role of brain insulin receptor in control of body weight and reproduction. Science, 2000: 289: 2122-2125.

15. Müller TD, Nogueiras R, Andermann ML, Andrews ZB, Anker SD, et al. Ghrelin. Mol Metab, 2015:4:437-460.

16. Strader AD, Woods SC. Gastrointestinal Hormones and Food Intake. Gastroenterology, 2005:128:175-191.

17. Wren AM, Bloom SR. Gut Hormones and Appetite Control. Gastroenterology, 2007:132:2116-2130.

18. Näslund E, Hellström PM. Appetite signaling: from gut peptides and enteric nerves to brain. Physiol Behav, 2007:10: 92:256-262.

19. Côté CD, Zadeh-Tahmasebi M, Rasmussen BA, Duca FA, Lam TK. Hormonal signaling in the gut. J Biol Chem, 2014: 289: 11642-11649.

20. Nergård BJ, Lindqvist A, Gislason HG, Groop L, Ekelund M, Wierup N, Hedenbro JL. Mucosal glucagon-like peptide-1 and gastric inhibitory polypeptide cell numbers in the super-obese human foregut after gastric bypass. Surg Obes Relat Dis, 2015: doi: 10.1016/j.soard.2015.03.021.

21. Hayes MR, Mietlicki-Baase EG, Kanoski SE, De Jonghe BC. Incretins and amylin: neuroendocrine communication between the gut, pancreas, and brain in control of food intake and blood glucose. Annu Rev Nutr, 2014: 34:237-260.

22. Jordi J, Herzog B, Camargo SM, Boyle CN, Lutz TA, Verrey F. Specific amino acids inhibit food intake via the area postrema or vagal afferents. J Physiol, 2013: 591: 5611-21.

23. Sohn JW, Elmquist JK, Williams KW. Neuronal circuits that regulate feeding behavior and metabolism. Trends Neurosci, 2013: 36: 504-512.

24. Keen-Rhinehart E, Ondek K, Schneider JE. Neuroendocrine regulation of appetitive ingestive behavior. Front Neurosci, 2013: 7: Art 213: 1-11.

25. Sohn JW. Network of hypothalamic neurons that control appetite. BMB Rep, 2015: 48: 229-233.

26. Dutia R, Kim AJ, Modes M, Rothlein R, Shen JM, Tian YE, Ihbais J, Victory SF, Valcarce C, Wardlaw SL. Effects of AgRP inhibition on energy balance and metabolism in rodent models. PLoS One, 2013:8:1-17.

27. Cansell C, Denis RG, Joly-Amado A, Castel J, Luquet S. Arcuate AgRP neurons and the regulation of energy balance. Front Endocrinol (Lausanne), 2012: 3: Art 169:1-7.

28. Kim JD, Leyva S. Hormonal regulation of the hypothalamic melanocortin system. Front Physiol, 2014: 5: Art 480: 1-7.

29. Yanik T, Dominguez G, Kuhar MJ, Del Giudice EM, Loh YP. The Leu34Phe ProCART mutation leads to cocaine- and amphetamine-regulated transcript (CART) deficiency: a possible cause for obesity in humans. Endocrinology, 2006:147: 39-43.

30. Subhedar NK, Nakhate KT, Upadhya MA, Kokare DM. CART in the brain of vertebrates: circuits, functions and evolution. Peptides, 2014: 54: 108-130. 
31. Loh K, Herzog H, Shi YC. Regulation of energy homeostasis by the NPY system. Trends Endocrinol Metab, 2015: 26:125-35.

32. Zhang W, Bai S, Liu D, Cline MA, Gilbert ER. Neuropeptide Y promotes adipogenesis in chicken adipose cells in vitro. Comp Biochem Physiol A Mol Integr Physiol, 2015:181: 62-70.

33. Ulrich-Lai YM, Ryan KK. Neuroendocrine circuits governing energy balance and stress regulation: functional overlap and therapeutic implications. Cell Metab, 2014:19: 910-25.

34. Harris RB. Chronic and acute effects of stress on energy balance: are there appropriate animal models? Am J Physiol Regul Integr Comp Physiol, 2015: 308: R250R265.

35. Einfeld SL, Smith E, McGregor IS, Steinbeck K, Taffe J, Rice LJ, Horstead SK, Rogers N, Hodge MA, Guastella AJ. A double-blind randomized controlled trial of oxytocin nasal spray in Prader Willi syndrome. Am J Med Genet A, 2014:164:22322239.

36. Ott V, Finlayson G, Lehnert H, Heitmann B, Heinrichs M, Born J, Hallschmid M. Oxytocin reduces reward-driven food intake in humans. Diabetes, 2013: 62:34183425.

37. Klockars A, Levine AS, Olszewski PK. Central Oxytocin and Food Intake: Focus on Macronutrient-Driven Reward. Front Endocrinol (Lausanne), 2015: 6: Art 65: 1-7.

38. Roseberry AG, Stuhrman K, Dunigan AI. Regulation of the mesocorticolimbic and mesostriatal dopamine systems by -melanocyte stimulating hormone and agouti-related protein. Neurosci Biobehav Rev, 2015, doi: 10.1016/j.neubiorev.2015.06.020.

39. Messina G, Dalia C, Tafuri D, Monda V, Palmieri F, Dato A, Russo A, De Blasio S, Messina A, DeLuca V, Chieffi S, Monda M. Orexin-A controls sympathetic activity and eating behavior. Front Psychol, 2014: 5: Art 997: 1.7.

40. Adamantidis A, de Lecea L. A role for Melanin-Concentrating Hormone in learning and memory. Peptides, 2009: 30: 2066.2070.

41. Sherwood A, Holland PC, Adamantidis A, Johnson AW. Deletion of Melanin Concentrating Hormone Receptor-1 disrupts overeating in the presence of food cues. Physiol Behav, 2015: doi: 10.1016/j.physbeh.2015.05.03

42. Konadhode RR, Pelluru D, Shiromani PJ. Neurons containing orexin or melanin concentrating hormone reciprocally regulate wake and sleep. Front Syst Neurosci, 2015: 8: Art 244: 1-9.

43. Pelluru D, Konadhode R, Shiromani PJ. MCH neurons are the primary sleep-promoting group. Sleep, 2013: 36: 1779-1781.

44. Chung S, Parks GS, Lee C, Civelli O. Recent updates on the melanin-concentrating hormone $(\mathrm{MCH})$ and its receptor system: lessons from MCH1R antagonists. J Mol Neurosci, 2011: 3: 115-121.

45. McCutcheon JE. The role of dopamine in the pursuit of nutritional value. Physiol Behav, 2015: doi: 10.1016/j.physbeh.2015.05.003.

46. Jordi J, Herzog B, Camargo SM, Boyle CN, Lutz TA, Verrey. F Specific amino acids inhibit food intake via the area postrema or vagal afferents. J Physiol, 2013: doi: 10.1113/jphysiol.2013.258947. 
47. Colombo G, Agabio R, Diaz G, Lobina C, Reali R, Gessa GL. Appetite suppression and weight loss after the cannabinoid antagonist SR 141716. Life Sci, 1998: 63:113117.

48. Ravinet Trillou C, Arnone M, Delgorge C, Gonalons N, Keane P, Maffrand JP, Soubrie P. Anti-obesity effect of SR141716, a CB1 receptor antagonist, in diet-induced obese mice. Am J Physiol Regul Integr Comp Physiol, 2003: 284:R345-353.

49. Doyon C, Denis RG, Baraboi ED, Samson P, Lalonde J, Deshaies Y, Richard D. Effects of rimonabant (SR141716) on fasting-induced hypothalamic-pituitary-adrenal axis and neuronal activation in lean and obese Zucker rats. Diabetes, 2006:55:3403-3410.

50. Wagner JD, Zhang L, Kavanagh K, Ward GM, Chin JE, Hadcock JR, Auerbach BJ, Harwood HJ Jr. A selective cannabinoid-1 receptor antagonist, PF-95453, reduces body weight and body fat to a greater extent than pair-fed controls in obese monkeys. J Pharmacol Exp Ther, 2010: 335: 103-113.

51. Holzer P, Farzi A. Neuropeptides and the microbiota-gut-brain axis. Adv Exp Med Biol, 2014: 817:195-219.

52. Clarke G, Stilling RM, Kennedy PJ, Stanton C, Cryan JF, Dinan TG. Minireview: Gut microbiota: the neglected endocrine organ. Mol Endocrinol, 2014:1221-1238. 